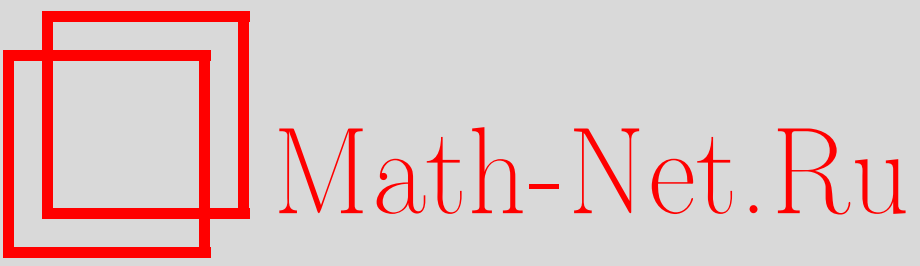

Л. Г. Шагалова, Кусочно линейная функция цены дифференциальной игры с простой динамикой и интегрально-терминальным функционалом платы, Итоги науки и техн. Сер. Соврем. мат. и ее прил. Темат. обз., 2019, том 168, 114-122

DOI: https://doi.org/10.36535/0233-6723-2019-168-114-122

Использование Общероссийского математического портала Math-Net.Ru подразумевает, что вы прочитали и согласны с пользовательским соглашением

http://www.mathnet.ru/rus/agreement

Параметры загрузки:

IP: 54.81 .137 .203

26 апреля 2023 г., $17: 35: 45$ 


\title{
КУСОЧНО ЛИНЕЙНАЯ ФУНКЦИЯ ЦЕНЫ ДИФФЕРЕНЦИАЛЬНОЙ ИГРЫ С ПРОСТОЙ ДИНАМИКОЙ И ИНТЕГРАЛЬНО-ТЕРМИНАЛЬНЫМ ФУНКЦИОНАЛОМ ПЛАТЫ
}

\author{
(C) 2019 г. $\quad$ Л. Г. ШАГАЛОВА
}

\begin{abstract}
АннотАция. Рассматривается антагонистическая дифференциальная игра двух лиц с динамикой, описываемой дифференциальным уравнением с простыми движениями, и интегральнотерминальным функционалом платы. В такой игре существует функция цены, которая является обобщенным (минимаксным/вязкостным)решением соответствующего уравнения ГамильтонаЯкоби. Для случая, когда терминальная функция и гамильтониан кусочно линейны, а размерность фазового пространства равна двум, предлагается конечный алгоритм точного построения функции цены. Алгоритм сводится к последовательному решению элементарных задач, возникающих в определенном порядке. Кусочно линейная функция цены дифференциальной игры формируется в результате склейки кусочно линейных решений элементарых задач. Удобным средством представления таких функций являются структурные матрицы.
\end{abstract}

Ключевые слова: дифференциальная игра, простое движение, функция цены, уравнение Гамильтона-Якоби, обобщенное решение, минимаксное решение, алгоритм.

\section{PIECEWISE-LINEAR PRICE FUNCTION \\ OF A DIFFERENTIAL GAME WITH SIMPLE DYNAMICS AND INTEGRAL-TERMINAL PRICE FUNCTIONAL}

\author{
(c) 2019 L. G. SHAGALOVA
}

\begin{abstract}
In this paper, we consider an antagonistic differential game of two persons with dynamics described by a differential equation with simple motions and an integral-terminal board functional. In this game, there exists a price function, which is a generalized (minimax or viscous) solution of the corresponding Hamilton-Jacobi equation. For the case where the terminal function and the Hamiltonian are piecewise linear and the dimension of the phase space is equal to 2 , we propose a finite algorithm for the exact construction of the price function. The algorithm is reduced to the sequential solution of elementary problems arising in a certain order. The piecewise linear price function of a differential game is constructed by gluing piecewise linear solutions of elementary problems. Structural matrices are a convenient tool of representing such functions.
\end{abstract}

Keywords and phrases: differential game, simple motion, price function, Hamilton-Jacobi equation, generalized solution, minimax solution, algorithm.

AMS Subject Classification: 49N70, 49N75, 91A05, 91A24

1. Введение. Антагонистические дифференциальные игры-раздел математической теории оптимального управления, в котором задачи управления исследуются в случае противоположных

Работа выполнена при поддержке Российского фонда фундаментальных исследований (проект № 17-01-00074) и Министерства образования и науки Российской Федерации (проект № 02.А03.21.0006). 
интересов управляющих системой субъектов, называемых игроками. Динамика системы описывается дифференциальным уравнением, содержащим управляющие векторы, которыми распоряжаются игроки. Существуют различные формализации дифференциальной игры.

В настоящей работе игровая задача рассматривается в рамках позиционной формализации, введенной в работах Н. Н. Красовского и А. И. Субботина (см. [2,11]). Для выбора своего управления каждый игрок может использовать лишь текущую информацию о состоянии системы. Первый игрок стремится минимизировать на движениях системы заданный функционал платы, цель второго игрока противоположна: он максимизирует этот функционал. В рассматриваемом случае подход к решению дифференциальной игры заключается в поиске функции цены. Функция цены заданному начальному состоянию системы ставит в соответствие оптимальный, одинаковый для каждого из игроков, гарантированный результат в игре: наилучшее гарантированное значение функционала платы. Зная функцию цены, можно построить оптимальные управления игроков по принципу обратной связи.

При исследовании дифференциальных игр, как правило, возникают нелинейные уравнения в частных производных первого порядка. В $[4,14]$ указано, что функция цены является минимаксным решением уравнения Гамильтона-Якоби, соответствующего рассматриваемой дифференциальной игре. При этом понятие обобщенного минимаксного решения эквивалентно понятию вязкостного решения, введенного М. Дж. Крэндаллом и П.-Л. Лионсом (см. [9]).

Таким образом, задача построения функции цены дифференциальной игры может быть сведена к решению дифференциального уравнения первого порядка. При этом для нелинейных уравнений Гамильтона-Якоби не существует универсального метода решения, и далеко не всегда удается получить решение в аналитической форме. Поэтому актуальными направлениями исследований являются разработка вычислительных методов и анализ структуры кусочно гладких минимаксных решений.

При локальной аппроксимации кусочно гладкой функции цены дифференциальной игры с динамикой общего вида можно использовать решение дифференциальной игры с простой динамикой, зависящей только от управлений игроков и не зависящей от фазового вектора. Несмотря на простоту динамики, решения таких игр известны лишь в некоторых частных случаях, а в общем случае нахождение решений также является нелегкой задачей. Поэтому исследование дифференциальных игр с простыми движениями имеет и самостоятельный интерес. Для исследования таких игр привлекаются геометрические методы, выпуклый и негладкий анализ. Дифференциальные игры с простыми движениями исследовались многими авторами; в частности, можно отметить работы $[1,6,12,13]$.

В настоящей работе представлен конечный алгоритм построения точного минимаксного решения уравнения Гамильтона-Якоби, соответствующего дифференциальной игре с простыми движениями и интегрально-терминальной платой, в случае двумерного фазового пространства и кусочно линейных входных данных. Представленные результаты обобщают результаты, полученные в $[5,7,15]$.

2. Постановка задачи. Рассматривается следующая позиционная дифференциальная игра двух лиц. Динамика управляемой системы имеет вид

$$
\dot{x}=u(t)+v(t), \quad t \in[0, \vartheta], \quad x \in \mathbb{R}^{n}, \quad u(t) \in P \subset \mathbb{R}^{n}, \quad v(t) \in Q \subset \mathbb{R}^{n}
$$

здесь $t$ - время, $\vartheta$ - заданный момент окончания игры, $x$-фазовый вектор, $u(\cdot)$ и $v(\cdot)-$ формируемые по принципу обратной связи управления первого и второго игроков соответственно. Предполагается, что множества $P$ и $Q$ - компакты.

Пусть $\left(t_{0}, x_{0}\right) \in[0, \vartheta] \times \mathbb{R}^{n}$ - начальная позиция. На движениях управляемой системы $(1)$, соответствующих начальной позиции, задан интегрально-терминальный функционал платы

$$
I=I\left(t_{0}, x_{0}, u(\cdot), v(\cdot)\right)=\sigma(x(\vartheta))+\int_{t_{0}}^{\vartheta} g(u(\tau), v(\tau)) d \tau
$$


где функция $\sigma: \mathbb{R}^{n} \rightarrow \mathbb{R}$ предполагается липшицевой, а функция $g: P \times Q \rightarrow \mathbb{R}$ - непрерывной. Первый игрок выбором своего управления стремится минимизировать плату, второй игрокмаксимизировать.

Предположим, что для рассматриваемой дифференциальной игры выполнено следующее условие:

$$
\min _{u \in P} \max _{v \in Q}[\langle s, u+v\rangle+g(u, v)]=\max _{v \in Q} \min _{u \in P}[\langle s, u+v\rangle+g(u, v)]=H(s), \quad s \in \mathbb{R}^{n},
$$

где $\langle s, f\rangle$ обозначает скалярное произведение векторов $s$ и $f$. Функцию $H(\cdot)$, определенную равенством (3), будем называть гамильтонианом дифференциальной игры (1), (2).

Известно (см. $[2,11])$, что при выполнении условия (3) для любой начальной позиции $\left(t_{0}, x_{0}\right) \in$ $[0, \vartheta] \times \mathbb{R}^{n}$ существует цена $\omega\left(t_{0}, x_{0}\right)$ игры. Таким образом, существует функция цены $\omega:$ $[0, \vartheta] \times \mathbb{R}^{n} \rightarrow \mathbb{R}$. Однако нахождение функции цены - весьма нелегкая задача, для решения которой не существует универсального метода. Отметим, что в случае, когда подынтегральная функция $g(\cdot)$ тождественно равна нулю, т.е. функционал платы является терминальным, задача существенно упрощается. В частности, если одна из функций $H(\cdot)$ или $\sigma(\cdot)$ является выпуклой или вогнутой, для функции цены можно, используя известные формулы Пшеничного-Сагайдак (см. [3]) и Хопфа-Лакса (см. $[8,10])$, выписать явные формулы. Также в случае не обязательно выпуклых кусочно линейных функций $H(\cdot)$ и $\sigma(\cdot)$, когда размерность $n$ фазового пространства равна двум, а терминальная плата положительно однородна, т.е. функция $\sigma(\cdot)$ удовлетворяет условию

$$
\sigma(\lambda x)=\lambda \sigma(x), \quad x \in \mathbb{R}^{n}, \quad \lambda \in \mathbb{R}, \quad \lambda>0,
$$

для построения функции цены можно использовать конечный алгоритм (см. $[5,7,15])$. Целью настоящей работы является обобщение этого алгоритма на случай ненулевой подынтегральной функции $g(\cdot)$.

3. Цена игры как минимаксное решение уравнения Гамильтона-Якоби. В этом разделе приведены используемые далее для разработки алгоритма построения функции цены дифференциальной игры (1)-(3) сведения из $[4,14]$ и факты, которые несложно получить из этих сведений.

Функция цены $\omega:[0, \vartheta] \times \mathbb{R}^{n} \rightarrow \mathbb{R}$ совпадает с минимаксным решением следующей задачи Коши:

$$
\begin{gathered}
\frac{\partial \omega(t, x)}{\partial t}+H\left(\frac{\partial \omega(t, x)}{\partial x}\right)=0, \quad t \leqslant \vartheta, \quad x \in \times \mathbb{R}^{n}, \\
\omega(\vartheta, x)=\sigma(x), \quad x \in \mathbb{R}^{n} .
\end{gathered}
$$

Минимаксное решение задачи (5), (6) существует и единственно. Далее будем предполагать, что терминальная функция $\sigma(\cdot)$ является положительно однородной, т.е. удовлетворяет условию (4). Гамильтониан $H(\cdot)$ определен равенством (3) и не является положительно однородным.

Определим функции

$$
\begin{aligned}
& H^{*}(s, r)=\left\{\begin{array}{ll}
|r| H\left(\frac{s}{|r|}\right) & \text { при } r \neq 0, \\
\lim _{r \downarrow 0} r H\left(\frac{s}{r}\right) & \text { при } r=0,
\end{array} \quad(s, r) \in \mathbb{R}^{n} \times \mathbb{R},\right. \\
& \sigma^{\sharp}(x, y)=\sigma(x)+y, \quad x \in \mathbb{R}^{n}, \quad y \in \mathbb{R} .
\end{aligned}
$$

Предполагаем, что предел в (7) существует.

Рассмотрим задачу Коши для уравнения Гамильтона-Якоби с положительно однородным относительно переменной $\bar{s}=(s, r)$ гамильтонианом $H^{*}(\cdot)$ :

$$
\begin{gathered}
\frac{\partial u(t, x, y)}{\partial t}+H^{*}\left(\frac{\partial u(t, x, y)}{\partial x}, \frac{\partial u(t, x, y)}{\partial y}\right)=0, \quad t \leqslant \vartheta, \quad(x, y) \in \mathbb{R}^{n} \times \mathbb{R}, \\
u(\vartheta, x, y)=\sigma^{\sharp}(x, y), \quad x \in \mathbb{R}^{n}, \quad y \in \mathbb{R} .
\end{gathered}
$$

Справедливо следующее утверждение. 
Теорема 1. Функиия $\omega(t, x)$ является минимаксным решением задачи (5), (6) тогда и только тогда, когда функиия $u(t, x, y)=\omega(t, x)+y$ является минимаксным решением задачи (9), (10).

Итак, задача нахождения функции цены с интегрально-терминальным функционалом платы сводится к решению уравнения Гамильтона-Якоби с положительно однородным гамильтонианом. При этом размерность фазового пространства увеличивается на единицу.

Если гамильтонан $H^{*}(\cdot)$ удовлетворяет условию Липшица, для минимаксного решения $u(t, x, y)$ имеет место соотношение

$$
u(t, x, y)=(\vartheta-t) u\left(0, \frac{x}{\vartheta-t}, \frac{y}{\vartheta-t}\right), \quad x \in \mathbb{R}^{n}, \quad y \in \mathbb{R} .
$$

Используя соотношение (11), можно заменить задачу (9), (10) редуцированной задачей нахождения функции

$$
\varphi(x, y)=u(0, x, y) \quad x \in \mathbb{R}^{n}, \quad y \in \mathbb{R} .
$$

Функция $\varphi(\cdot)$ является минимаксным решением следующего уравнения в частных производных первого порядка:

$$
H^{*}\left(\frac{\partial \varphi(x, y)}{\partial x}, \frac{\partial \varphi(x, y)}{\partial y}\right)+\left\langle\frac{\partial \varphi(x, y)}{\partial x}, x\right\rangle+\frac{\partial \varphi(x, y)}{\partial y} \cdot y-\varphi(x, y)=0, \quad x \in \mathbb{R}^{n}, \quad y \in \mathbb{R},
$$

которое рассматривается наряду с предельным соотношением

$$
\lim _{\alpha \downarrow 0} \alpha \varphi\left(\frac{x}{\alpha}, \frac{y}{\alpha}\right)=\sigma^{\sharp}(x, y), \quad x \in \mathbb{R}^{n}, \quad y \in \mathbb{R} .
$$

Минимаксное решение уравнения (13) - непрерывная функция, удовлетворяющая паре дифференциальных неравенств. Эти неравенства можно записать различными по форме, но эквивалентными по существу способами. Здесь удобно выписать эти неравенства в следующем виде:

$$
\begin{array}{llll}
H^{*}(l, m)+\langle l, x\rangle+m \cdot y \leqslant \varphi(x, y), & x \in \mathbb{R}^{n}, & y \in \mathbb{R}, & (l, m) \in D^{-} \varphi(x, y), \\
H^{*}(l, m)+\langle l, x\rangle+m \cdot y \geqslant \varphi(x, y), & x \in \mathbb{R}^{n}, & y \in \mathbb{R}, & (l, m) \in D^{+} \varphi(x, y),
\end{array}
$$

где множества $D^{-} \varphi(x, y)$ и $D^{+} \varphi(x, y)$ - субдифференциал и супердифференциал функции $\varphi(\cdot)$ в точке $(x, y)$ соответственно.

4. Алгоритм построения функции цены. В случае, когда размерность фазового пространства равна двум, а терминальная функция $\sigma(\cdot)$ и подынтегральная функция $g(\cdot)$ кусочно линейны, функция $\omega(\cdot)$ цены дифференциальной игры (1)-(3) является кусочно линейной и может быть построена точно. Опишем алгоритм построения функции $\varphi(\cdot)$, зная которую, можно получить функцию $\omega(\cdot)$ с помощью соотношения (11) и теоремы 1.

4.1. Представление предельной функиии. Пусть

$$
\begin{gathered}
y_{+}=\max \{0 ; y\}, \quad y_{-}=\min \{0 ; y\}, \quad y \in \mathbb{R}, \\
\sigma_{+}(x)=\max \{0 ; \sigma(x)\}, \quad \sigma_{-}(x)=\min \{0 ; \sigma(x)\}, \quad x \in \mathbb{R}^{n}, \\
\sigma_{+}^{\sharp}(x, y)=\sigma_{+}(x)+y_{+}, \quad \sigma_{-}^{\sharp}(x, y)=\sigma_{-}(x)+y_{-}, \quad x \in \mathbb{R}^{n}, \quad y \in \mathbb{R} .
\end{gathered}
$$

Нетрудно заметить, что предельная функция $\sigma^{\sharp}(\cdot)$ в (8) может быть представлена в виде

$$
\sigma^{\sharp}(x, y)=\sigma_{+}^{\sharp}(x, y)+\sigma_{-}^{\sharp}(x, y), \quad x \in \mathbb{R}^{n}, \quad y \in \mathbb{R} .
$$

При этом для решения $\varphi(\cdot)$ задачи $(13),(14)$ справедливо представление

$$
\varphi(x, y)=\varphi_{+}(x, y)+\varphi_{-}(x, y), \quad x \in \mathbb{R}^{n}, \quad y \in \mathbb{R},
$$

где $\varphi_{+}(\cdot)$ и $\varphi_{-}(\cdot)$ - решения задачи $(13),(14)$, соответствующие предельным функциям $\sigma_{+}^{\sharp}(\cdot)$ и $\sigma_{-}^{\sharp}(\cdot)$ соответственно. При описанных ниже предположениях построения функций $\varphi_{+}(\cdot)$ и $\varphi_{-}(\cdot)$ не отличаются по существу. 
5. Предположения. Алгоритм разработан при следующих предположениях.

A1. Подынтегральная функция $g(\cdot)$ имеет вид

$$
g(u, v)=g_{1}(u)+g_{2}(v), \quad u \in \mathbb{R}^{2}, \quad v \in \mathbb{R}^{2},
$$

где $g_{1}: \mathbb{R}^{2} \rightarrow \mathbb{R}$ и $g_{2}: \mathbb{R}^{2} \rightarrow \mathbb{R}$ - непрерывные кусочно линейные функции, склеенные из конечного числа линейных функций. Таким образом, их сумма $g(\cdot)$ тоже является непрерывной кусочно линейной функцией.

A2. Множества $P$ и $Q$ - многогранники. Из (3) следует, что гамильтониан $H(\cdot)$ дифференциальной игры (1)-(3) также кусочно линеен и склеивается из конечного числа линейных функций

$$
H^{i}(s)=\left\langle h^{i}, s\right\rangle+p^{i}, \quad i \in \overline{1, n_{H}}, \quad h^{i} \in \mathbb{R}^{2}, \quad p^{i} \in \mathbb{R}, \quad s \in \mathbb{R}^{2} .
$$

A3. Функция $\sigma(\cdot)$ положительно однородна (удовлетворяет условию (4)) и кусочно линейна, т.е. сформирована с помощью склейки конечной совокупности линейных функций

$$
\sigma^{i}(x)=\left\langle s^{i}, x\right\rangle, \quad i \in \overline{1, n_{\sigma}}, \quad s^{i} \in \mathbb{R}^{2}, \quad x \in \mathbb{R}^{2} .
$$

Введем обозначение

$$
Z=\left\{s^{i} \mid i \in \overline{1, n_{\sigma}}\right\} .
$$

Кроме того, в силу представлений (17), (18) без ограничения общности можно считать, что функция $\sigma(\cdot)$ неотрицательна:

$$
\sigma(x) \geqslant 0, \quad x \in \mathbb{R}^{2},
$$

и рассмотреть алгоритм построения функции $\varphi(\cdot)$, соответствующей предельной функции

$$
\sigma^{\sharp}(x, y)=\sigma(x)+y_{+}, \quad x \in \mathbb{R}^{2}, \quad y \in \mathbb{R}
$$

6. Простые кусочно линейные функции. Для разработки алгоритма полезным является использованное в $[5,15]$ понятие простой кусочно линейной функции (ПКЛФ). Основное свойство ПКЛФ заключается в следующем. Если функция $\psi: \mathbb{R}^{2} \supset D \rightarrow \mathbb{R}$ является ПКЛФ, тогда для любой точки $x_{*} \subset D$ существует окрестность $O_{\varepsilon}\left(x_{*}\right)$, в которой $\psi(\cdot)$ имеет одно из трех возможных представлений:

$$
\begin{gathered}
\psi(x)=\left\langle s_{i}, x\right\rangle+h_{i}, \\
\psi(x)=\max \left\{\left\langle s_{i}, x\right\rangle+h_{i},\left\langle s_{j}, x\right\rangle+h_{j}\right\}, \\
\psi(x)=\min \left\{\left\langle s_{i}, x\right\rangle+h_{i},\left\langle s_{j}, x\right\rangle+h_{j}\right\} .
\end{gathered}
$$

Здесь $s_{i}$ и $s_{j}$ - векторы из $\mathbb{R}^{2}$, а $h_{i}$ и $h_{j}$ - числа. Таким образом, область определения ПКЛФ не содержит точек, в малой окрестности которых склеиваются три или более линейных функций.

Для формального определения ПКЛФ используются структурные матрицы. Не будем приводить здесь строгое определение, отметим лишь, что структурная матрица содержит информацию обо всех линейных функциях, формирующих соответствующую ПКЛФ. Зная структурную матрицу, можно вычислить значение ПКЛФ в каждой точке ее области определения.

Отметим также, что при выполнении условия $\mathbf{A 3}$, неотрицательная функция $\sigma: \mathbb{R}^{2} \longrightarrow \mathbb{R}$ является ПКЛФ в области $\mathbb{R}^{2} \backslash 0$, где символом 0 обозначен нулевой вектор. На рис. 1 и 2 представлены примеры поведения линий уровня функции $\sigma$.

7. Элементарные задачи. Алгоритм построения функции $\varphi(\cdot)$ заключается, по существу, в последовательном решении элементарных задач, которые возникают в определенном порядке.

Пусть

$$
\varsigma^{\sharp+}(x, y)=\max \{\langle a, x\rangle+y,\langle b, x\rangle+y\}, \quad \varsigma^{\sharp-}(x, y)=\min \{\langle a, x\rangle+y,\langle b, x\rangle+y\},
$$

где $a, b, x$ - векторы из $\mathbb{R}^{2}, y \in \mathbb{R}$. 


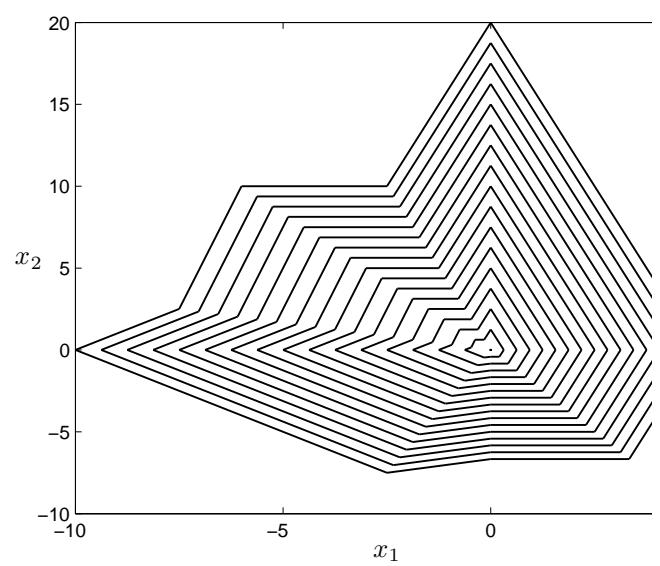

Рис. 1

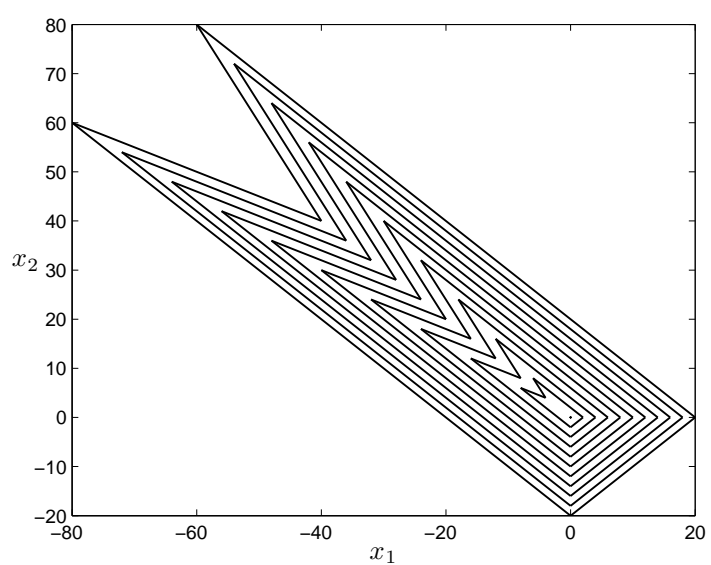

Рис. 2

Задачи 1 и 2. Пусть заданы некоторые линейно независимые векторы $a$ и $b$. В задаче 1 (задаче 2) требуется построить минимаксное решение задачи (13), (14), (20) при $\sigma^{\sharp}=\varsigma^{\sharp+}$ (соответственно, при $\left.\sigma^{\sharp}=\varsigma^{\sharp-}\right)$.

Поскольку функция $\varsigma^{\sharp+}$ выпукла, а функция $\varsigma^{\sharp-}$ вогнута, можно получить явные формулы для решений задач 1 и 2 . Можно показать, что решениями этих задач являются функции

$$
\phi^{+}(x, y)=\max _{l \in[a, b]} \phi_{l}(x, y), \quad \phi^{-}(x, y)=\min _{l \in[a, b]} \phi_{l}(x, y),
$$

где

$$
[a, b]=\{\lambda a+(1-\lambda) b \mid \lambda \in[0,1]\}, \quad \phi_{l}(x, y)=\langle l, x\rangle+y+H(l) .
$$

Первый этап алгоритма построения решения $\varphi(\cdot)$ задачи $(13),(14),(23)$ заключается в последовательном решении задач 1 и 2 и склейке из этих решений. Конкретные задачи, которые необходимо решить, определяются функцией $\sigma(\cdot)$.

Дальнейшее построение решения заключается в решении элементарных задач другого типа.

Пусть $\bar{s}=\left(s_{1}, s_{2}, s_{3}\right) \in \mathbb{R}^{3}$. Введем обозначение

$$
\varphi_{\bar{s}}(x, y)=\langle s, x\rangle+s_{3} \cdot y+H^{*}(\bar{s}), \quad x \in \mathbb{R}^{2}, \quad y \in \mathbb{R},
$$

где вектор $s \in \mathbb{R}^{2}$ образован из первых двух компонент вектора $\bar{s}, s=\left(s_{1}, s_{2}\right)$. Отметим, что если $s_{3}=1$, то $H^{*}(\bar{s})=H(s)$ и $\varphi_{\bar{s}}(x, y)=\phi_{s}(x, y)$.

Для заданного множества $M$ обозначим его замыкание символом $c l M$, а границу-символом $\partial M$.

Задачи 3 и 4. Рассмотрим линейно независимые векторы $\bar{a}=\left(a_{1}, a_{2}, a_{3}\right) \in \mathbb{R}^{3}$ и $\bar{b}=\left(b_{1}, b_{2}, b_{3}\right) \in$ $\mathbb{R}^{3}$ и число $r>0$. Пусть

$$
\begin{array}{cl}
\varphi^{*}(x, y)=\max \left\{\varphi_{\bar{a}}(x, y), \varphi_{\bar{b}}(x, y)\right\}, & \varphi_{*}(x, y)=\min \left\{\varphi_{\bar{a}}(x, y), \varphi_{\bar{b}}(x, y)\right\}, \\
G^{*}=\left\{(x, y) \in \mathbb{R}^{3} \mid \varphi^{*}(x, y)<r\right\}, & G_{*}=\left\{(x, y) \in \mathbb{R}^{3} \mid \varphi_{*}(x, y)<r\right\} .
\end{array}
$$

В задаче 3 требуется построить непрерывную функцию $\varphi^{0}: \operatorname{cl} G^{*} \rightarrow \mathbb{R}$, являющуюся в области $G^{*}$ минимаксным решением уравнения в частных производных (УЧП) первого порядка (13) и удовлетворяющую соотношениям

$$
\varphi^{0}(x, y)<r \forall(x, y) \in G^{*} ; \quad \varphi^{0}(x, y)=r \forall(x, y) \in \partial G^{*} .
$$

В задаче 4 требуется построить непрерывную функцию $\varphi_{0}: \operatorname{cl} G_{*} \rightarrow \mathbb{R}$, являющуюся в области $G_{*}$ минимаксным решением уравнения (13) и удовлетворяющую соотношениям

$$
\varphi_{0}(x, y)<r \forall(x, y) \in G_{*} ; \quad \varphi_{0}(x, y)=r \forall(x, y) \in \partial G_{*} .
$$


Решением задачи 3 является функция

$$
\varphi^{0}(x, y)=\max _{\bar{s}} \varphi_{\bar{s}}(x, y) \quad \text { при } \quad \bar{s} \in S_{r}(\bar{a}, \bar{b}),
$$

где

$$
\begin{gathered}
S_{r}(\bar{a}, \bar{b})=\left\{\bar{s} \in \operatorname{con}(\bar{a}, \bar{b}) \mid\left\langle s, w_{0}\right\rangle+H^{*}(\bar{s})=r\right\}, \\
\operatorname{con}(\bar{a}, \bar{b})=\{\lambda \bar{a}+\mu \bar{b} \mid \lambda \geqslant 0, \mu \geqslant 0\},
\end{gathered}
$$

а точка $w_{0} \in \mathbb{R}^{2}$ - решение системы двух линейных уравнений

$$
\left\langle a, w_{0}\right\rangle+H^{*}(\bar{a})=r, \quad\left\langle b, w_{0}\right\rangle+H^{*}(\bar{b})=r .
$$

Компоненты векторов $a \in \mathbb{R}^{2}$ и $b \in \mathbb{R}^{2}$ совпадают с первыми двумя компонентами векторов $\bar{a}$ и $\bar{b}$ соответственно.

Решением задачи 4 в случаях, возникающих при построении решения $\varphi(\cdot)$ задачи (13), (14), (23), является функция

$$
\varphi_{0}(x, y)=\min _{\bar{s}} \varphi_{\bar{s}}(x, y) \quad \text { при } \quad \bar{s} \in S_{r}(\bar{a}, \bar{b}) .
$$

8. Основной результат. Обозначим символом $\Omega$ множество точек из $\mathbb{R}^{3}$, в которых гамильтониан $H^{*}: \mathbb{R}^{3} \rightarrow \mathbb{R}$ недифференцируем. Пусть 0 - нулевой вектор в $\mathbb{R}^{3}$. По множеству $Z \subset \mathbb{R}^{2}$ (см. (21)) векторов, формирующих функцию $\sigma$, определим множество

$$
Z^{\natural}=\left\{\bar{s}=\left(s_{1}, s_{2}, s_{3}\right) \in \mathbb{R}^{3} \mid s=\left(s_{1}, s_{2}\right) \in Z, s_{3}=1\right\} \subset \mathbb{R}^{3} .
$$

Сформулируем основной результат работы.

Теорема 2. Пусть выполнены условия А2-A3. Тогда справедливы следующие утверждения.

A. Решение $\varphi(\cdot)$ задачи (13), (14), (23) - неотрицательная кусочно линейная функиия, образованная с помощью склейки линейных функций

$$
\varphi_{\bar{s}}(x, y)=\langle s, x\rangle+s_{3} \cdot y+H^{*}(\bar{s}), \quad \bar{s} \in L,
$$

где можество L состоит из конечного числа элементов, и

$$
Z^{\natural} \subset L, \quad\left(L \backslash Z^{\natural}\right) \subset(\Omega \cup 0) .
$$

В. Для любого $y_{*} \in \mathbb{R}$ функиия $\varphi\left(x, y_{*}\right)$ в области $\left\{x \in \mathbb{R}^{2} \mid \varphi\left(x, y_{*}\right)>0\right\}$ образована с помощью склейки конечного числа простых кусочно линейных функиий.

Доказательство теоремы 2 следует из описанного выше алгоритма. Неотрицательность функции $\varphi(\cdot)$ обусловлена неотрицательностью функции $\sigma(\cdot)$, вид $(24)$ линейных функций, формирующих решение, обусловлен конкретными элементарными задачами, возникающими в ходе его построения.

Для доказательства того, что построенная в результате алгоритма функция $\varphi(\cdot)$ является минимаксным решением уравнения (13), необходимо проверить выполнение неравенств (15), (16). В точках, где $\varphi(\cdot)$ совпадает с решением какой-либо из рассмотренных выше элементарных задач, эти неравенства выполнены. Таким образом, следует проверить выполнение неравенств (15), (16) на поверхностях склейки решений различных элементарных задач, формирующих функцию $\varphi(\cdot)$. При этом если поверхность Г склейки не принадлежит никакой из областей определения решений элементарных задач, существует такое число $r \geqslant 0$, что $\Gamma \subset\left\{(x, y) \in \mathbb{R}^{2} \times \mathbb{R} \mid \varphi(x, y)=r\right\}$.

Пусть $\left(x_{*}, y_{*}\right) \in \Gamma$. Если существует окрестность $O_{\varepsilon}\left(x_{*}, y_{*}\right)$, в которой функция $\varphi$ линейна, неравенства (15), (16) выполняются. Действительно, в этом случае существует такой вектор $\bar{s}^{*}=$ $\left\{s^{*}, s_{3}^{*}\right\}, s^{*} \in \mathbb{R}^{2}, s_{3}^{*} \in \mathbb{R}$, что

$$
\varphi(x, y)=\varphi_{\bar{s}^{*}}=\left\langle s^{*}, x\right\rangle+s_{3}^{*} \cdot y+H^{*}\left(\bar{s}^{*}\right), \quad\left(x_{*}, y_{*}\right) \in O_{\varepsilon}\left(x_{*}, y_{*}\right) .
$$

Функция $\varphi$ дифференцируема в $O_{\varepsilon}\left(x_{*}, y_{*}\right)$, и $D^{-} \varphi\left(x_{*}, y_{*}\right)=D^{+} \varphi\left(x_{*}, y_{*}\right)=\bar{s}^{*}$, и в точке $\left(x_{*}, y_{*}\right)$ выполняются неравенства (15), (16).

Рассмотрим случай, когда в точке $\left(x_{*}, y_{*}\right) \in \Gamma$ происходит склейка двух линейных функций. Из алгоритма следует, что в этом случае возможны две ситуации. В первой для любого $\varepsilon>0$ в окрестности $O_{\varepsilon}\left(x_{*}, y_{*}\right)$ можно указать точку $\left(x_{\varepsilon}, y_{\varepsilon}\right)$, в которой функция $\varphi$ склеивается из тех же 
линейных функций и является решением какой-либо элементарной задачи, причем $\varphi\left(x_{\varepsilon}, y_{\varepsilon}\right)>r$. Очевидно, что в этой ситуации

$$
D^{-} \varphi\left(x_{*}, y_{*}\right)=D^{-} \varphi\left(x_{\varepsilon}, y_{\varepsilon}\right), \quad D^{+} \varphi\left(x_{*}, y_{*}\right)=D^{+} \varphi\left(x_{\varepsilon}, y_{\varepsilon}\right) .
$$

Поскольку в точке $\left(x_{\varepsilon}, y_{\varepsilon}\right)$ неравенства $(15),(16)$ выполняются, в силу непрерывности получим, что они выполнены и в точке $\left(x_{*}, y_{*}\right)$.

Во второй ситуации в некоторой окрестности $O_{\varepsilon}\left(x_{*}, y_{*}\right)$ точки $\left(x_{*}, y_{*}\right)$ функция $\varphi$ имеет вид

$$
\varphi(x, y)=\max \left\{\varphi_{\bar{a}}(x, y), \varphi_{\bar{b}}(x, y)\right\},
$$

где векторы $\bar{a}, \bar{b}$ из $\mathbb{R}^{3}$ связаны равенством $\bar{b}=\mu \bar{a}, \mu>0$, причем вектор $\bar{a}$ ненулевой. Имеем $D^{+} \varphi\left(x_{*}, y_{*}\right)$,

$$
D^{-} \varphi\left(x_{*}, y_{*}\right)=\left\{\bar{l} \in \mathbb{R}^{3} \mid \bar{l}=\lambda \bar{a}, \lambda \in[0,1]\right\} \cup\left\{\bar{l} \in \mathbb{R}^{3} \mid \bar{l}=\lambda \bar{b}, \lambda \in[0,1]\right\} .
$$

Так как функции $\varphi_{\bar{a}}$ и $\varphi_{\bar{b}}$ положительно однородны и

$$
\varphi\left(x_{*}, y_{*}\right)=\varphi_{\bar{a}}\left(x_{*}, y_{*}\right)=\varphi_{\bar{b}}\left(x_{*}, y_{*}\right)=r \geqslant 0,
$$

получаем, что и в этой ситуации неравенства (15), (16) выполняются.

Рассмотрим, наконец, случай, когда точка $\left(x_{*}, y_{*}\right)$ является узловой, т.е. в ней происходит склейка $n(n \geqslant 3)$ линейных функций

$$
\varphi_{\bar{a}^{p}}(x, y)=\left\langle a^{p}, x\right\rangle+a_{3}^{p} \cdot y+H^{*}\left(\bar{a}^{p}\right), \quad p=1, \ldots, n .
$$

Функция $\varphi$ не дифференцируема в точке $\left(x_{*}, y_{*}\right)$, поэтому одно из множеств $D^{-} \varphi\left(x_{*}, y_{*}\right)$, $D^{+} \varphi\left(x_{*}, y_{*}\right)$ пусто. Примем для определенности, что $D^{+} \varphi\left(x_{*}, y_{*}\right)=\varnothing$. Если при этом $D^{-} \varphi\left(x_{*}, y_{*}\right)=\varnothing$, неравенства (15), (16), очевидно, выполняются.

Предположим, что $D^{-} \varphi\left(x_{*}, y_{*}\right) \neq \varnothing$. Обозначим символом $\varphi_{\varepsilon}(\cdot)$ сужение функции $\varphi$ на такую достаточно малую выпуклую окрестность $O_{\varepsilon}\left(x_{*}, y_{*}\right)$, что в этой окрестности функция $\varphi$ склеивается только из функций (25). Можно показать, что

$$
D^{-} \varphi\left(x_{*}, y_{*}\right)=D^{-} \tilde{\varphi}_{\varepsilon}\left(x_{*}, y_{*}\right) \text {, }
$$

где $\tilde{\varphi}_{\varepsilon}(\cdot)$ - выпуклая оболочка функции $\varphi_{\varepsilon}$. Таким образом, $D^{-} \varphi\left(x_{*}, y_{*}\right)$ является ограниченным замкнутым выпуклым множеством. При этом если $\bar{l}^{*} \in D^{-} \varphi\left(x_{*}, y_{*}\right)$, то существуют такие векторы $\bar{a} \in \mathbb{R}^{3}, \bar{b} \in \mathbb{R}^{3}$, что

$$
\bar{l}^{*} \in[\bar{a}, \bar{b}]=\{(1-\lambda) \bar{a}+\lambda \bar{b} \mid \lambda \in[0,1]\},
$$

и для любого $n=1,2, \ldots$ существует такая точка $\left(x_{n}, y_{n}\right) \in O_{\varepsilon_{n}}\left(x_{*}, y_{*}\right)$, что $D^{-} \varphi\left(x_{n}, y_{n}\right)=[a, b]$. Здесь $\varepsilon_{n}=\varepsilon / n$. Точки $\left(x_{n}, y_{n}\right)$ не являются узловыми, поэтому в них выполнено неравенство (15). В частности,

$$
H^{*}\left(\bar{l}^{*}\right)+\left\langle l, x_{n}\right\rangle+l_{3} \cdot y_{n} \leqslant \varphi\left(x_{n}, y_{n}\right) .
$$

Переходя в (26) к пределу при $n \rightarrow \infty$, получим

$$
H^{*}\left(\bar{l}^{*}\right)+\left\langle l, x_{*}\right\rangle+l_{3} \cdot y_{*} \leqslant \varphi\left(x_{*}, y_{*}\right),
$$

что доказывает выполнение неравенства (15). Неравенство (16) выполнено в силу пустоты супердифференциала.

Случай, когда $D^{+} \varphi\left(x_{*}, y_{*}\right) \neq \varnothing, D^{+} \varphi\left(x_{*}, y_{*}\right)=\varnothing$ рассматривается аналогично. Таким образом, построенная в результате алгоритма функция $\varphi(\cdot)$ является минимаксным решением уравнения (13).

\section{СПИСОК ЛИТЕРАТУРЫ}

1. Камнева Л. В., Пацко В. С. Построение максимального стабильного моста в играх с простыми движениями на плоскости// Тр. ИММ УрО РАН. - 2014. - 20, № 4. - С. 128-142.

2. Красовский Н. Н., Субботин А. И. Позиционные дифференциальные игры. - М.: Наука, 1974.

3. Пшеничный Б. Н., Сагайдак М. И. О дифференциальных играх с фиксированным временем// Кибернетика. - 1970. - № 2. - С. 54-63.

4. Субботин А. И. Минимаксные неравенства и уравнения Гамильтона-Якоби. - М.: Наука, 1991. 
5. Субботин А. И., Шагалова Л. Г. Кусочно линейное решение задачи Коши для уравнения ГамильтонаЯкоби // Докл. РАН. - 1992. - 325, № 5. - С. 144-148.

6. Ухоботов В. И., Цеунова И. В. Дифференциальная игра с простым движением// Вестн. Челябинск. гос. ун-та. - 2009. - 11. - С. 84-96.

7. Шагалова Л. Г. Кусочно линейная функция цены дифференциальной игры с простыми движениями// Вестн. Тамбов. ун-та. Сер. Естеств. и техн. науки. - 2007. - 12, № 4. - С. 564-565.

8. Bardi M., Evans L. On Hopf's formulas for solutions of Hamilton-Jacobi equations// Nonlin. Anal. Th. Meth. Appl. — 1984. — 8, № 11. - P. 1373-1381.

9. Crandall M. G., Lions P. L. Viscosity solutions of Hamilton-Jacobi equations// Trans. Am. Math. Soc. 1983. - 377, № 1. - P. 1-42.

10. Hopf E. Generalized solutions of nonlinear equations of first order// J. Math. Mech. — 1965. — 14, № 6 . - P. 951-973.

11. Krasovskii N. N., Subbotin A. I. Game-Theoretical Control Problems. - New York: Springer-Verlag, 1988.

12. Pachter M., Yavin Y. Simple-motion pursuit-evasion differential games. Part 1. Stroboscopic strategies in collision-course guidance and proportional navigation// J. Optim. Theory Appl. — 1986. — 51, № 1. — P. 95-127.

13. Petrosjan L. A. Differential games of pursuit. — Singapore: World Scientific, 1993.

14. Subbotin A. I. Generalized Solutions of First Order PDEs. The Dynamical Optimization Perspective. Boston: Birkhäuser, 1995.

15. Shagalova L. G. A piecewise linear minimax solution of the Hamilton-Jacobi equation// IFAC Proc. Vols. - 1998. - 31, № 13. - P. 193-197.

Шагалова Любовь Геннадьевна

Институт математики и механики им. Н. Н. Красовского УрО РАН

Уральский федеральный университет имени первого Президента России Б. Н. Ельцина

E-mail: shag@imm.uran.ru 